\title{
Erratum to: Blood parasites in reptiles imported to Germany
}

\author{
Ursula Halla • Rüdiger Korbel • Frank Mutschmann • \\ Monika Rinder
}

Published online: 23 November 2014

(C) Springer-Verlag Berlin Heidelberg 2014

Erratum to: Parasitology Research

DOI 10.1007/s00436-014-4149-5

First and last names of all authors were regretfully published inverted. The correct names with addresses are listed below.

U. Halla $(*) \cdot$ R. Korbel $\cdot$ M. Rinder

Klinik für Vögel, Reptilien, Amphibien und Zierfische, Zentrum für klinische Tiermedizin, Tierärztliche Fakultät, Ludwig-Maximilians Universität München, Sonnenstr. 18, 85764 Oberschleißheim, Germany

e-mail: Ursula.Halla@vogelklinik.vetmed.uni-muenchen.de

F. Mutschmann

Exomed-Institut für veterinärmedizinische Betreuung niederer Wirbeltiere und Exoten, Erich-Kurz-Str. 7, 10319 Berlin, Germany

The online version of the original article can be found at http://dx.doi.org/ 10.1007/s00436-014-4149-5.

U. Halla $(\bowtie) \cdot$ R. Korbel $\cdot$ M. Rinder

Klinik für Vögel, Reptilien, Amphibien und Zierfische, Zentrum für

klinische Tiermedizin, Tierärztliche Fakultät, Ludwig-Maximilians

Universität München, Sonnenstr. 18, 85764 Oberschleißheim,

Germany

e-mail: Ursula.Halla@vogelklinik.vetmed.uni-muenchen.de

F. Mutschmann

Exomed-Institut für veterinärmedizinische Betreuung niederer

Wirbeltiere und Exoten, Erich-Kurz-Str. 7, 10319 Berlin, Germany 